\title{
Malignant Transformation of Monostotic Fibrous Dysplasia after Surgery: A Case Report
}

\author{
Abigail R. Tud ${ }^{1}$, Cesar D. Dimayuga ${ }^{1}$
}

\section{Abstract}

Introduction: Fibrous dysplasia (FD) is a benign lesion characterized by replacement of normal bone with abnormal connective tissue. It occurs in monostotic or polyostotic forms, with a rare but proven potential for malignant transformation. Symptoms of acute pain, rapid swelling, or an enlarging mass should increase suspicion for possible sarcomatous change. Complete surgical resection is the mainstay of treatment, and chemotherapy is recommended to improve survival.

Case Report: A 52-year-old male presented with a painful, enlarging mass on the right proximal thigh 2 years after undergoing plate fixation of the distal femur for a pathologic fracture secondary to monostotic FD. Diagnostic imaging revealed signs of recurrence, and core needle biopsy revealed aggressive features suggestive of malignant transformation. Following surgical resection and chemotherapy, the outcome has been uneventful without evidence of recurrence or metastasis at 4-year post-operation.

Conclusion: Malignant transformation in monostotic FD is rare. Symptom exacerbation should increase the suspicion for sarcomatous change and prompt the need for diagnostic imaging as well as histologic confirmation.

Keywords: Fibrosarcoma, fibrous dysplasia, malignant transformation, secondary sarcoma.

\section{Introduction}

Fibrous dysplasia (FD) is a benign bone lesion accounting for $5-10 \%$ of all benign bone tumors, with a rare but proven potential for malignant transformation $[1,2,3]$. It may present with monostotic or polyostotic involvement, with the monostotic variant more commonly affecting the craniofacial bones, ribs, and long bones [3, 4, 5]. Malignant transformation in the monostotic form is rare, with an estimated frequency of $0.5 \%[3,5,6]$. Secondary sarcomas developing from FD include osteosarcoma, fibrosarcoma, chondrosarcoma, and undifferentiated pleomorphic sarcoma $[1,5]$. Our report presents the clinical features and diagnostic findings of a patient with malignant transformation of monostotic FD.

\section{Case Report}

A 50-year-old male presented with a pathologic fracture of the distal femur (Fig. 1A-D). Core needle biopsy before plate fixation (Fig. 2A and B) and intraoperative specimens confirmed tissue diagnosis of FD.

The patient's post-operative course was unremarkable, and he returned to premorbid function without need for assistance. Two years post-surgery, the patient reported severe pain over the right thigh during ambulation, with note of a firm swelling on the anterior aspect. Repeat X-rays showed an increase in size of the previous lesion, while magnetic resonance imaging (MRI) revealed marrow signals suggestive of tumor progression.

At this point, core needle biopsy findings remained consistent with FD (Fig. 3), and the patient was managed symptomatically. To augment pain relievers, monthly denosumab administration at $120 \mathrm{mg}$ was initiated according to recommendations from literature [7]. The patient's symptoms improved until the 6th month when there was note of increased pain and development of a palpable mass at the right proximal thigh.

Subsequent radiographs showed poorly marginated, osteolytic meta-diaphyseal lesions with permeative borders, in addition to the existing lesions at the previous operative site (Fig. 4A and C). Evidence of neocorticalization, a break on the posterolateral cortex, and the presence of a soft tissue mass were also noted. Repeat MRI revealed an extensive extraosseous soft tissue component surrounding the proximal third of the right femur (Fig. 5A-C), measuring $11.5 \mathrm{~cm} \times 15.4 \mathrm{~cm}$. The lesion was heterogenous in signal intensity, predominantly hyperintense to muscle on all sequences, and continuous across the cortical break on the posterolateral aspect with the intra-osseous component.

Repeat core needle biopsy now revealed dysplastic spindle cells exhibiting malignant features and absence of osteoid. Ablative surgery was advised, and a modified hemipelvectomy was carried out with an unremarkable post-operative course.

Gross specimens showed an encapsulated doughy mass measuring $17 \mathrm{~cm}$ in length, with circumference of $39 \mathrm{~cm}$. An irregularly
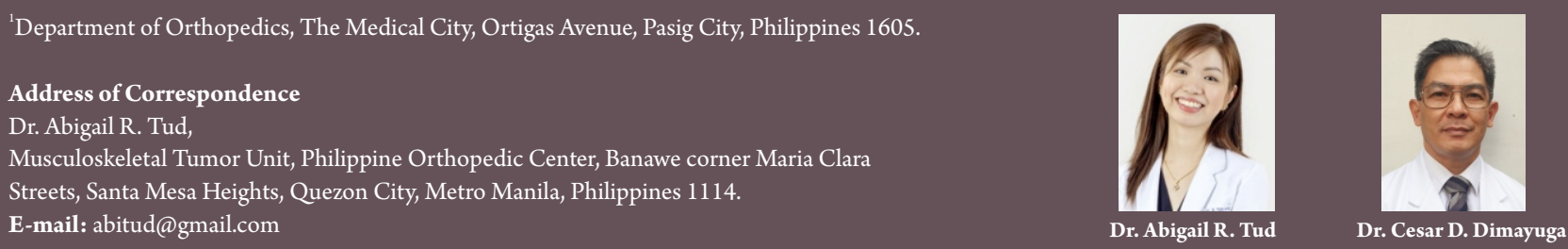

Submitted: 21/2/2021; Review: 23/2/2021; Accepted: 15/4/ 2021; Published:15/5/ 2021

(C) 2021 by Journal of Bone and Soft Tissue Tumors | Available on www.jbstjournal.com| DOI:10.13107/jbst.2021.v07i02.46 This is an Open Access aticle distributed under the terms of the Creatiive Commons Attbution Non-Commercial-Share Alike 4.0 License (http:/eativecommons.org/licenses/by-nc-sa/4.0) which allows others to remix, tweak, and build upon the work non-commercially as long as appropriate credit is given and the new creation are licensed under the identical terms. 


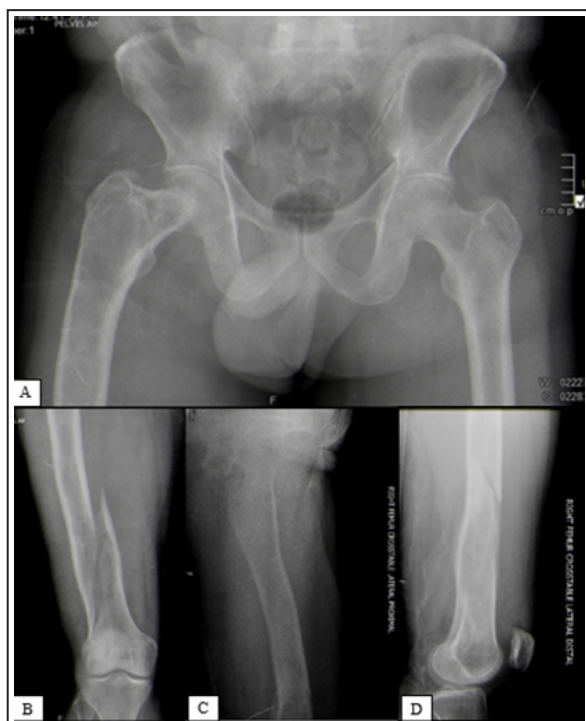

Figure 1: Anteroposterior view of the pelvis $(\mathrm{A})$ and the right distal femur (B), and lateral views (C and D) of the right femora shaft before initial fixation. Radiographs show "ground glass" appearance with endosteal scalloping and a classic "Shepherd's Crook" deformity over the right proximal femur. A pathologic fracture can be appreciated at the distal third of the femoral shaft.

shaped opening on the posterolateral aspect of the proximal femur was consistent with the cortical break documented on MRI. Histologic examination of this area revealed highly pleomorphic, hyperchromatic spindle cells interspersed with cells with round to polygonal nuclei, a high nuclear to cytoplasmic ratio, and scant basophilic cytoplasm. Mitotic count was at 80 mitoses per ten high-power fields, with evidence of vascular invasion. These features confirmed the diagnosis of a high-grade fibrosarcoma

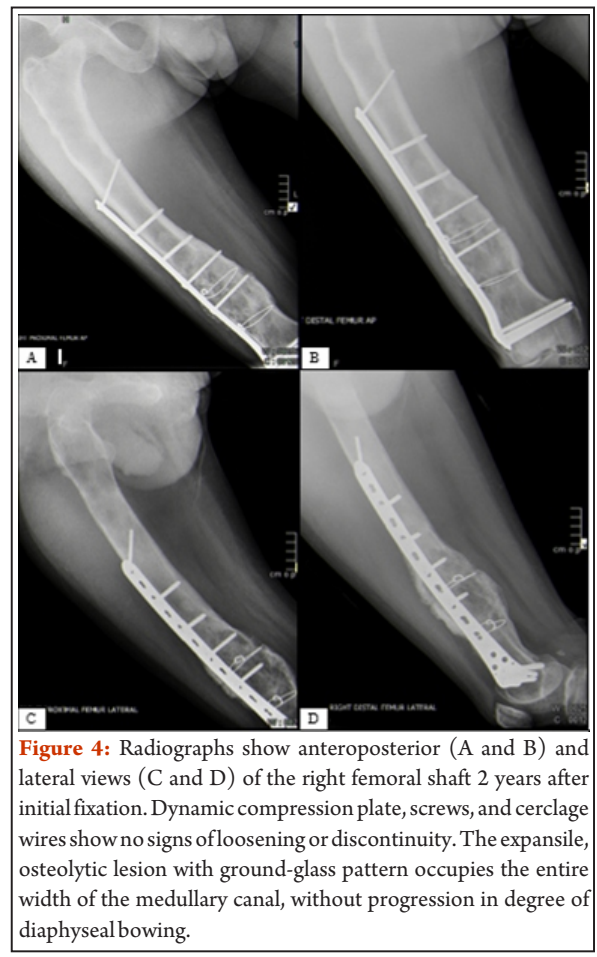

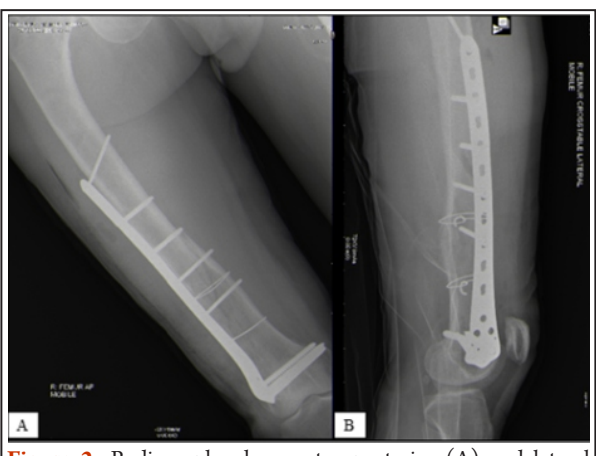

Figure 2: Radiographs show anteroposterior (A) and lateral views (B) of the right femoral shaft immediately after plate fixation for pathologic fracture. Dynamic compression plate, screws, and cerclage wires are now noted, with adequate fracture reduction and restoration of length, axis, and rotation.

\section{(Fig. 6A).}

Sections taken from the middle third of the femur showed the clear transition from benign to sarcomatous features (Fig. 6B and 7): Benign fibroblasts with bony spicules characteristic of FD transitioning into malignant fibroblasts interspersed with fibrous stroma and woven bone. All surgical margins were negative following resection.

The patient completed six cycles of adjuvant chemotherapy based on National Comprehensive Cancer Network (NCCN) recommendations for fibrosarcoma (doxorubicin, ifosfamide, and mesna). He is currently 4-year post-surgery, without evidence of recurrence or metastatic disease as confirmed through positron emission tomography (PET) and chest computed tomography (CT) scans. While initially fitted with an artificial limb, the prostheses proved to be cumbersome for the patient and he has since opted to use modified elbow crutches to perform activities of daily living.

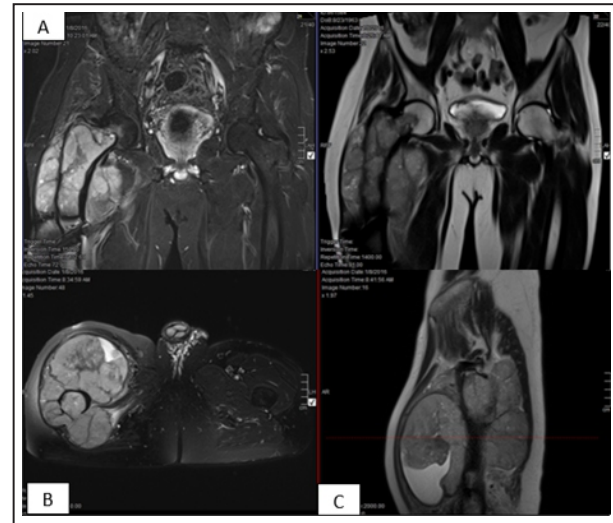

Figure 4: Representative magnetic resonance imaging cuts showing coronal (A), axial (B), and sagittal (C) sections of the tumor on T2 (left column) and T1-weighted imaging (right column). Note the heterogenous signals predominantly hyperintense to muscle, with low-signal, thin septations and welldefined cystic components more evident on the axial and sagittal cuts.

2| Journal of Bone and Soft Tissue Tumors

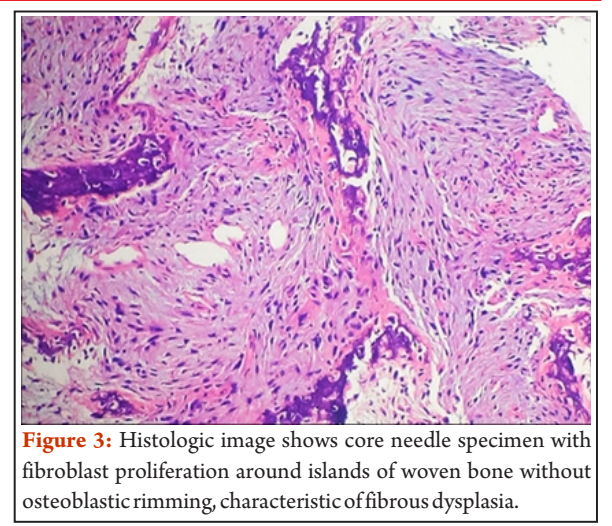

Discussion

Malignant tumor progression from underlying benign lesions is welldocumented but uncommon events [1]. The first case of secondary sarcoma arising in FD was reported in $1945[3,6]$. Since then, its potential for malignant transformation has been estimated at $1 \%$. At the Rizzoli Institute, secondary sarcoma arising from previously diagnosed FD accounted for just $0.26 \%$ of all treated malignant bone sarcomas from 1970 to 2005 [1]. This is consistent with earlier reports by Ruggieri et al. estimating the overall frequency of sarcoma in FD as $0.4-6.7 \%[6]$.

In patients with $\mathrm{FD}$, normal bone structure is replaced with abnormal connective tissue, characterized histologically by low to moderately cellular fibrous stroma, with irregularly shaped trabeculae of woven bone. The curvilinear bony trabeculae account for its typical histologic description resembling "Chinese characters" $[2,5]$.

FD shows no gender predilection and is usually diagnosed between the ages of 10 and 30 years following a pathologic fracture or incidental discovery. The mean age of sarcoma occurrence has been reported between 30 and 60 years, predominating among individuals above 40 years old $[3,6]$. Clinical presentation of acute pain, rapid swelling, or an enlarging mass should increase suspicion for possible malignant degeneration $[1,3]$. These features are consistent with disease presentation for our patient.

FD occurs in monostotic or polyostotic forms. The monostotic variant is more common, ranging from $70 \%$ to $80 \%$ of cases and commonly affects the craniofacial bones, ribs, and long bones. The polyostotic form occurs in around $20-30 \%$ of cases, with a higher predilection for the femur, tibia, craniofacial bones, pelvis, and ribs. This 


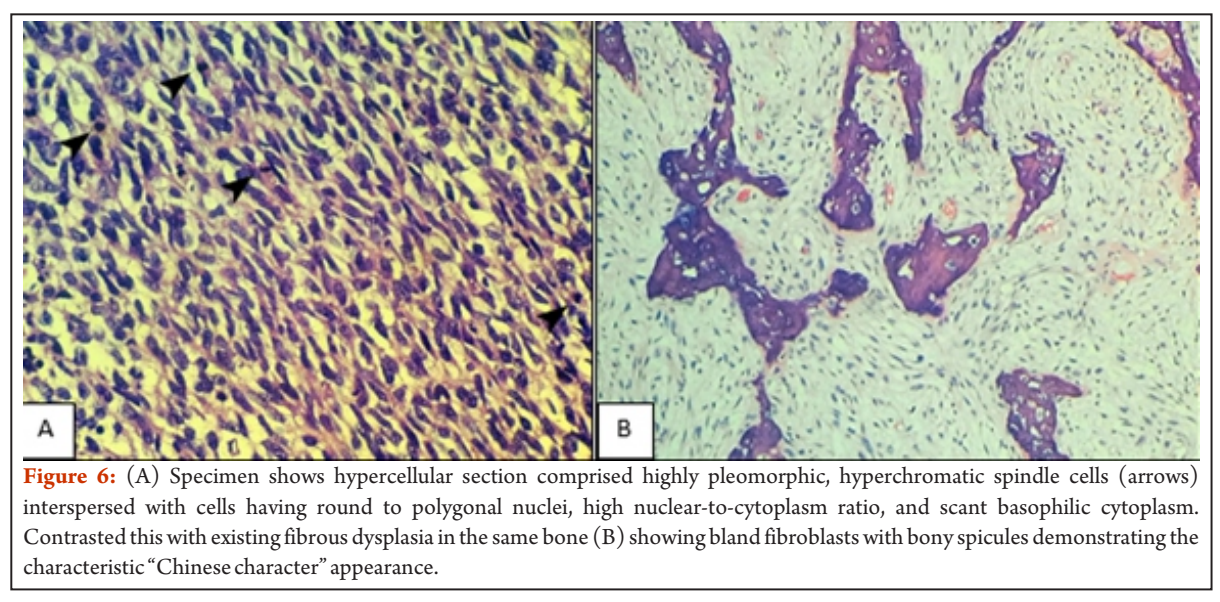

condition has been associated with a somatic gene mutation in the short arm of chromosome 20 (20q13), an area that codes for the $\alpha$-subunit on $\mathrm{G}$-protein receptors and is also present in several endocrine tumors [3, $4,5]$. A subset of patients with FD may in turn have associated endocrinologic abnormalities and café-au-lait spots: The characteristic triad for McCune-Albright syndrome (MAS). In a smaller percentage, fibro-osseous lesions may be present in the setting of multiple intramuscular myxomas, constituting features of Mazabraud syndrome (MS) [5].

While the exact pathogenesis of secondary sarcoma in FD remains undetermined, its frequency is increased in the polyostotic form, as well as in patients with MAS, MS, and a history of radiation exposure. Malignant transformation in the monostotic form is rare, with an estimated frequency of $0.5 \%[3,5,6]$. In a 2015 case series on ten patients, biopsy confirmed to have malignant

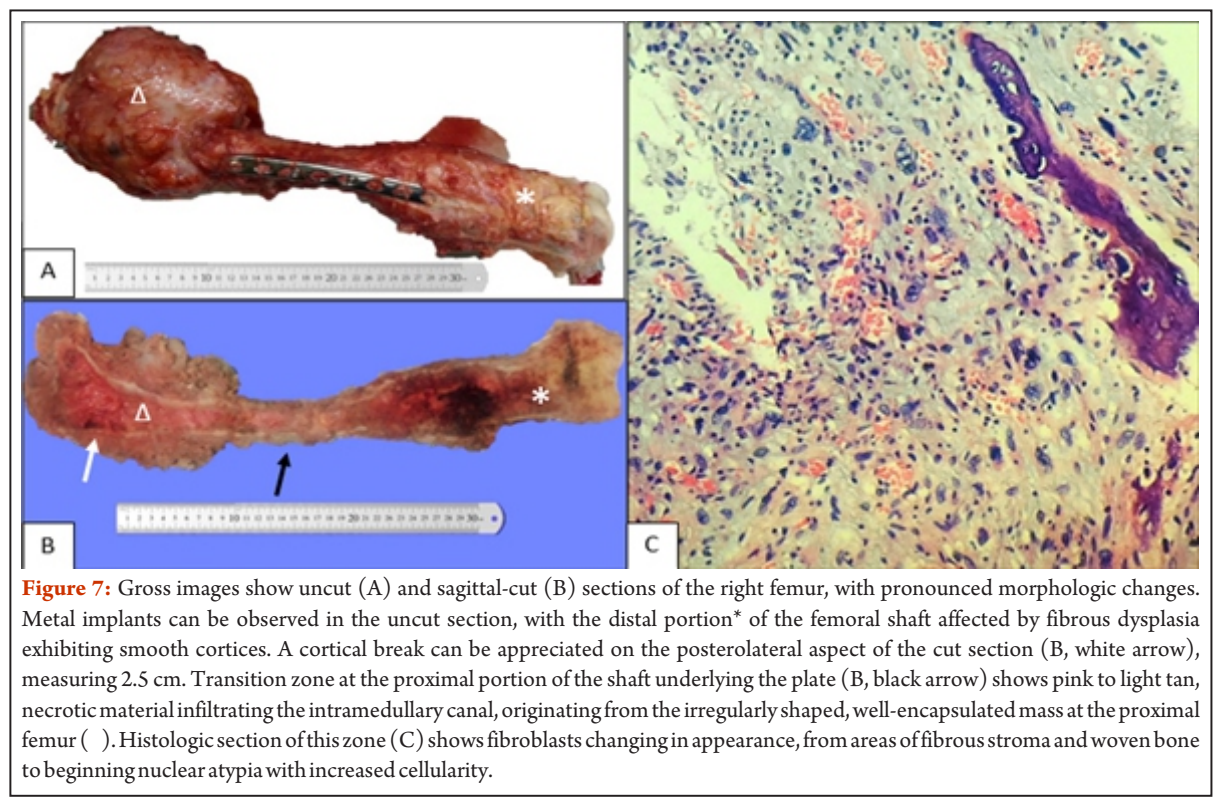

3| Journal of Bone and Soft Tissue Tumors curettage and bone grafting to ORIF for fractures, as a risk factor for the occurrence of secondary sarcoma. Thus far, however, there has been insufficient evidence to support any direct association between surgical intervention and malignant transformation $[1,3,6,8,9,10]$.

In FD, plain radiographs typically show areas of lucency with a "ground glass" pattern and areas of endosteal scalloping but otherwise no periosteal reaction. It is often associated with the characteristic "shepherd's crook" deformity of the proximal femur, secondary to coxa vara of the femoral neck, and increased curvature of the proximal femoral shaft $[2,5,8]$. In cases of secondary sarcoma, osteolytic lesions with signs of aggressiveness such as permeative margins, cortical break, and soft tissue extension were consistently found within or near areas previously affected by FD. Several studies have documented the superiority of CT scans at depicting cortical destruction and the presence of mineralization in such cases $[1,3]$. MRI has been recommended for delineation of bone marrow involvement as well as characterization of soft tissue masses. Signal enhancement, heterogenous signals, and soft tissue masses with extensive peritumoral/marrow edema have all been associated with malignant transformation. All of these findings were present in the MRI of our patient.

The prognosis for patients with malignant degeneration of FD is a function of timely diagnosis and prompt initiation of appropriate intervention. Complete surgical resection continues to be the mainstay of treatment, augmented by pre- or postoperative chemotherapy to improve local control as well as overall survival. The specific chemotherapy regimen depends on the histologic subtype of the secondary sarcoma present. The NCCN recommends two chemotherapy agents used in osteosarcoma (doxorubicin and cisplatin) for patients diagnosed with undifferentiated pleomorphic sarcoma and fibrosarcoma as first-line therapy [11]. Good pathologic response and survival rates have been reported, with median survival time at 63 months. The 5-year progression-free survival is at $56 \%$, with overall survival rate at $59 \%$ [ 3 , 11].

Our patient completed 6 cycles of doxorubicin, cisplatin, and mesna, with an 
unremarkable post-operative course. PET and chest CT scans done at regular intervals showed no signs of recurrence or metastasis and no evidence of polyostotic involvement. It must be stressed to patients, however, that the reported prognosis for secondary sarcomas in FD is poor; mortality rates as high as 54\% have been reported, highlighting the need for surveillance and continuous follow-up, especially within the first 2 years after resection $[6,8]$.

\section{Conclusion}

Malignant transformation in monostotic FD is rare, and recognition is dependent on an accurate clinical history. Acute and rapid symptom exacerbation should increase the suspicion for secondary sarcomatous change and prompt the need for diagnostic imaging. Radiologic features suggestive of malignant transformation include new areas of osteolysis, permeative borders, and evidence of a cortical break with soft tissue extension. MRI findings consistent with these features include heterogeneity, increased signal intensity on T2 imaging, and contrast enhancement. Histologic examination confirms the diagnosis of secondary sarcoma and will aid in surgical planning as well as selection of systemic treatment.

\section{References}

1. Picci P, Sieberova G, Alberghini M, Balladelli A, Vanel D, Hogendoorn $P C$, et al. Late sarcoma development after curettage and bone grafting of benign bone tumors. EurJ Radiol 2011;77:19-25.

2. Muthusamy S, Conway SA, Subhaeong TY, Temple HT. Locally aggressive fibrous dysplasia mimicking malignancy: A report of four cases and review of the literature. Clin Orthop Relat Res 2015;473:742-50.

3. Qu N, Yao W, Cui X, Zhang H. Malignant transformation in monostotic fibrous dysplasia: Clinical features, imaging features, outcomes in 10 patients, and review. Medicine (Baltimore) 2015;94:e369.

4. Mardekian SK, Tuluc M. Malignant sarcomatous transformation of fibrous dysplasia. Head Neck Pathol 2015;9:100-3.

5. Riddle ND, Bui MM. Fibrous dysplasia. Arch Pathol Lab Med 2013;137:821.

6. Ruggieri $P$, Sim FH, Bond JR, Unni KK. Malignancies in fibrous dysplasia. Cancer 1994;73:1411-24.

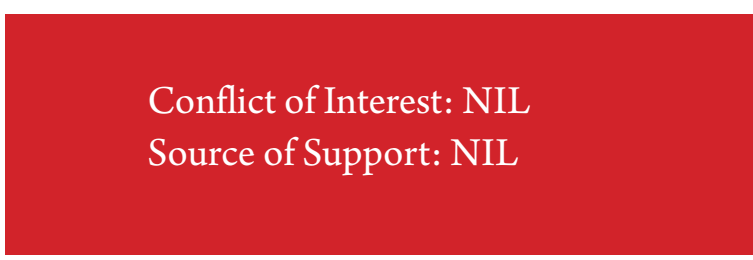

\section{How to Cite this Article}

Tud AR, Dimayuga CD| Malignant Transformation of Monostotic Fibrous Dysplasia after Surgery: A Case Report | Journal of Bone and Soft Tissue Tumors | May-August 2021;7(2): 1-4. 